Koenraad Verboven

\title{
Ce que negotiari et ses dérivés veulent dire
}

\author{
ANDREAU Jean \& CHANKOWSKI Véronique (edd.), Vocabulaire et expression de l'économie \\ dans le monde antique, Bordeaux, Ausonius, 2007, p. 89-118 \\ Please note that this is a preprint version before final revisions were \\ made. For reference purposes the print edition should be used
}

L'objectif de cette enquête est une étude socio-psychologique à travers les 'mots' et les concepts appartenant à la branche 'économique' de la famille de negotium. Le but est d'établir l'arrière plan social du choix des mots. Pourquoi est-ce qu'un auteur ou quelqu'un qui érige une inscription choisit tel ou tel mot et non pas un autre? Le langage des negotia ne se comprend pas seulement par les diverses significations matérielles possibles des termes. Les mots et surtout le formulaire servent aussi comme des signifiants de positions sociales (réelles, fictives, désirées ou réclamées) utilisés par l'auteur pour appliquer - le plus souvent inconsciemment - une classification sociale. Cette classification n'est pas une donnée immuable, mais un acte social de l'auteur inspiré par sa position et ses convictions personnelles. Ce n'est pas un acte arbitraire ; il reflète l'organisation du monde perçue par l'auteur ou dont la perception par les lecteurs est anticipée par l'auteur.

Mais avant d'étudier cette dynamique sociale derrière le langage des negotia, il nous faut dire un mot sur la méthode utilisée et les significations matérielles des mots.

\section{Méthodologie}

Cette étude repose sur une analyse de l'usage des mots et des expressions appartenant à la famille de negotium / negotiari utilisés dans un sens 'économique' (y compris la gestion des patrimoines) dans la totalité des inscriptions latines publiées et dans les textes latins non bibliques sur le cdrom \#5 du Packhard Humanities Institute ${ }^{1}$.

Le matériel épigraphique comprend 343 inscriptions où negotium ou l'un de ses dérivés sont utilisés, dont 326 où ils sont utilisés pour décrire des acteurs ou des activités économiques. Le PHI 5 cdrom fournit une liste de 38 auteurs latins qui utilisent au total 2210 fois le mot negotium ou l'un de ses dérivés. J'y ai ajouté le De viris illustribus, dont le texte est facilement repérable sur l'Internet et qui nous fournit encore 2 attestations. A partir de cette liste j'ai fait une sélection de 490 passages où negotium ou ses dérivés sont utilisés une ou plusieurs fois pour décrire des acteurs, des sujets ou des situations 'économiques'. Je ne prétends pas n'avoir manqué aucun passage, mais la sélection est à mon avis assez large et variée pour constituer un échantillon représentatif pour la période de Plaute jusqu'au IV ${ }^{\mathrm{e}}$ siècle p.C..

La répartition de negotium et ses dérivés dans la littérature latine est très inégale. Cicéron (621 fois) et les Digestes (771 fois) fournissent presque deux tiers de toutes les occurrences. Dans le cas des Digestes, cette fréquence est due à l'importance juridique de la gestion de patrimoines par des tiers (gestio / administratio negotiorum). Mais dans

\footnotetext{
${ }^{1}$ PHI CD Rom \#5.3. (1) Latin Texts (2) Bible Versions, Packhard Humanities Institute, 1991.
} 
le cas de Cicéron il s'agit simplement d'une préférence stylistique. Cicéron seul fournit environ $43 \%$ des attestations dans les textes non juridiques. La répartition des passages 'économiques' montre le même déséquilibre. Cicéron (146) et les Digestes (178) fournissent deux tiers de tous les passages, Cicéron fournit presque la moitié (47\%) de tous les passages dans les textes non juridiques.

\begin{tabular}{|lllll|}
\hline & \multicolumn{3}{l}{ attestations } & passages 'économiques \\
\hline Apuleius & 2212 & & 490 & \\
Asconius & 30 & $1,36 \%$ & 7 & $1,43 \%$ \\
Aulus Gellius & 2 & $0,09 \%$ & 1 & $0,20 \%$ \\
Bellum Africum & 55 & $2,49 \%$ & 3 & $0,61 \%$ \\
Bellum Alexandrinum & 5 & $0,23 \%$ & 3 & $0,61 \%$ \\
Caesar & 6 & $0,27 \%$ & 0 & $0,00 \%$ \\
Cato maior & 30 & $1,36 \%$ & 5 & $1,02 \%$ \\
Celsus & 4 & $0,18 \%$ & 0 & $0,00 \%$ \\
Cicero & 11 & $0,50 \%$ & 0 & $0,00 \%$ \\
Columella & $\mathbf{6 2 1}$ & $\mathbf{2 8 , 0 7 \%}$ & $\mathbf{1 4 6}$ & $\mathbf{2 9 , 8 0 \%}$ \\
De viris illustribus & 27 & $1,22 \%$ & 12 & $2,45 \%$ \\
Festus & 2 & $0,09 \%$ & 2 & $0,41 \%$ \\
Frontinus & 12 & $0,54 \%$ & 3 & $0,61 \%$ \\
Fronto & 8 & $0,36 \%$ & 3 & $0,61 \%$ \\
Gaius & 41 & $1,85 \%$ & 2 & $0,41 \%$ \\
Horace & 26 & $1,18 \%$ & 15 & $3,06 \%$ \\
Digestes & 11 & $0,50 \%$ & 5 & $1,02 \%$ \\
Iuvenalis & $\mathbf{7 7 1}$ & $\mathbf{3 4 , 8 6 \%}$ & $\mathbf{1 7 8}$ & $\mathbf{3 6 , 3 3 \%}$ \\
Livius & 2 & $0,09 \%$ & 0 & $0,00 \%$ \\
Martialis & 61 & $2,76 \%$ & 4 & $0,82 \%$ \\
Nepos & 5 & $0,23 \%$ & 3 & $0,61 \%$ \\
Petronius & 9 & $0,41 \%$ & 1 & $0,20 \%$ \\
Plinius maior & 11 & $0,50 \%$ & 6 & $1,22 \%$ \\
Plinius minor & 24 & $1,08 \%$ & 13 & $2,65 \%$ \\
Porphyrio & 26 & $1,18 \%$ & 2 & $0,41 \%$ \\
Ps.Varro & 14 & $0,63 \%$ & 1 & $0,20 \%$ \\
Q. Cicero & 4 & $0,18 \%$ & 1 & $0,20 \%$ \\
Quintilianus & 4 & $0,18 \%$ & 0 & $0,00 \%$ \\
Sallustius & 57 & $2,58 \%$ & 17 & $3,47 \%$ \\
Scriptores Historiae Augustae & 53 & $2,40 \%$ & 6 & $1,22 \%$ \\
Seneca jr & 15 & $0,68 \%$ & 5 & $1,02 \%$ \\
Seneca sr. & 88 & $3,98 \%$ & 13 & $2,65 \%$ \\
Servius & 9 & $0,41 \%$ & 4 & $0,82 \%$ \\
Suetonius & 39 & $1,76 \%$ & 1 & $0,20 \%$ \\
Tacitus & 39 & $1,76 \%$ & 8 & $1,63 \%$ \\
Valerius Maximus & 54 & $2,44 \%$ & 12 & $2,45 \%$ \\
Varro & 16 & $0,72 \%$ & 2 & $0,41 \%$ \\
Velleius Paterculus & 4 & $0,18 \%$ & 1 & $0,20 \%$ \\
Vitruvius & 11 & $0,50 \%$ & 2 & $0,41 \%$ \\
& 5 & $0,23 \%$ & 3 & $0,61 \%$ \\
\hline
\end{tabular}

Les inscriptions qui utilisent le vocabulaire des negotia dans un sens économique sont réparties de façon très inégale sur les différentes provinces et l'Italie. Les provinces gauloises, germaniques et la Britannia fournissent presque $32 \%$ des attestations. Les provinces du Danube et Dalmatia en fournissent un peu plus de $12 \%$. L'Italie sans 
Rome et sans Ostia-Portus fournit $17 \%$, Rome fournit presque $22 \%$ et Ostia-Portus à peu près $4 \%^{2}$. Les provinces espagnoles et africaines sont presque absentes de la documentation.

Cette répartition inégale témoigne de la différence entre les habitudes épigraphiques dans ces régions, plutôt que des différences sociales ou économiques éventuelles. L'absence d'indications professionnelles n'est pas remarquable, car la grande majorité des inscriptions funéraires, votives ou honorifiques, ne mentionnent aucune profession. L'épitaphe que Trimalcion prévoit pour son monument funèbre mentionne sa qualité de bienfaiteur et d'augustalis, mais non pas le fait qu'il avait fait fortune comme negotiator $^{3}$. Dans les provinces africaines et espagnoles, des attestations de collèges

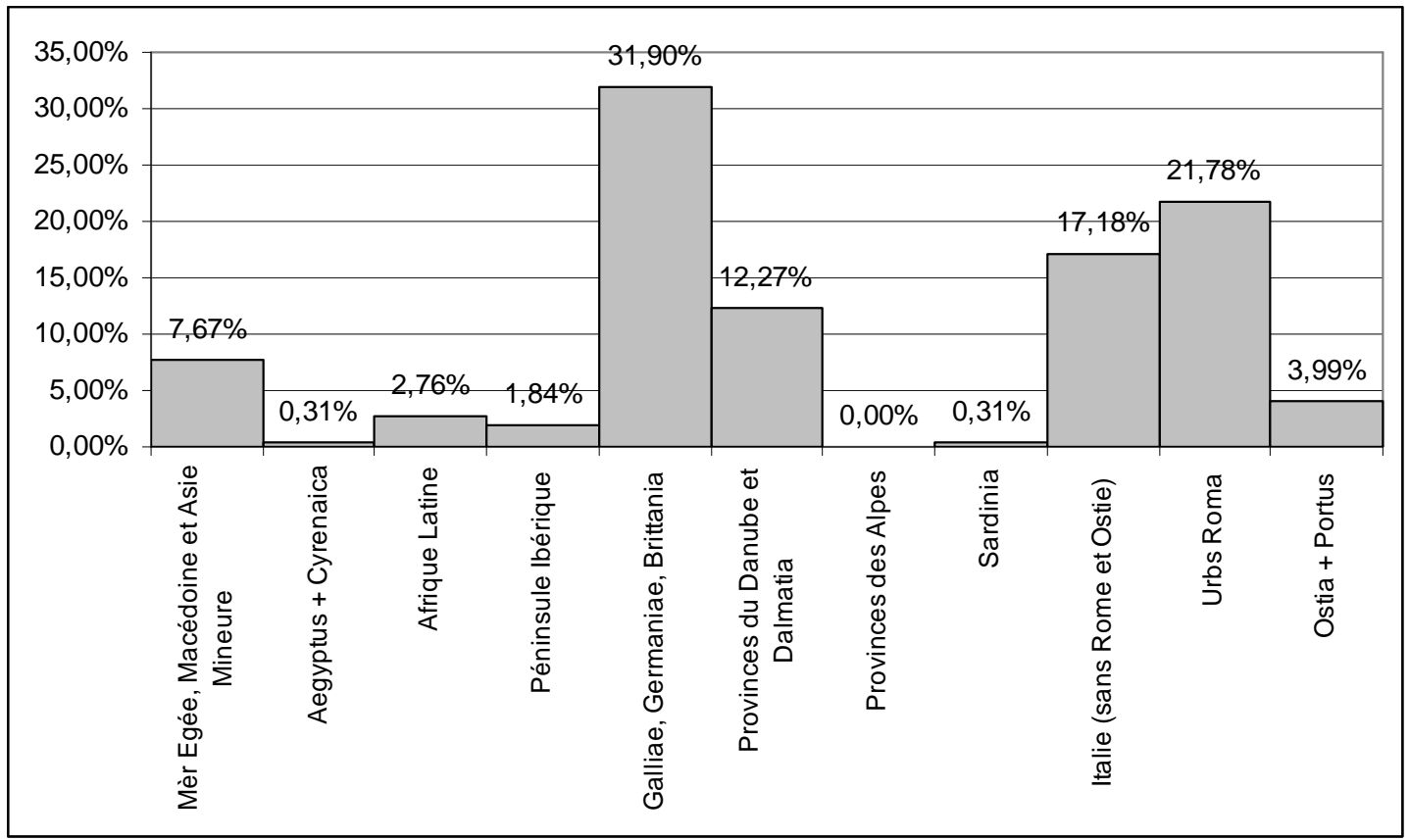

professionnels aussi sont plutôt rares.

La rareté du vocabulaire des negotia dans les inscriptions des provinces grecques ne nécessite guère d'explication ${ }^{4}$. L'abondance du matériel en Italie est relative, étant donné la richesse épigraphique de l'Italie. Mais l'abondance du matériel provenant des provinces du Nord-Ouest - plutôt pauvres en inscriptions - et la rareté du matériel provenant des provinces espagnoles et africaines - relativement riches en inscriptions est remarquable $\mathrm{e}^{5}$. En regardant de plus près, la concentration se situe surtout dans les provinces longeant le Rhin et dans la ville de Lyon. La Gallia Belgica et la Raetia

\footnotetext{
${ }^{2}$ Mais notez AE 1900, 178 = CIL, I ${ }^{2}, 836$ de Tarracina : cives Romani in Sicilia Panhormi qui/ negotiantur.

${ }^{3}$ Petr. 71

${ }^{4}$ A l'exception de $A E$ 1946, 230 (Amphipolis), toutes les inscriptions de provenance 'grecque' sont des inscriptions honorifiques offertes par des communautés de cives Romani / Italic(e)i qui negotiantur.

${ }^{5}$ Le chiffre élevé de Germania Inferior résulte en partie des inscriptions en honneur de Nehalennia, dont 14 parlent de negotiatores. Cf. Stuart \& Bogaers 2001
} 
semblent s'y conformer. Par contre, la Narbonnaise, l'Aquitaine et le reste de Gallia Lugdunensis, ainsi que Britannia fournissent beaucoup moins d'attestations. En Italie, les plus grandes concentrations se situent dans le Latium et Rome et au Nord, dans la Transpadana et la Venetia-Histria

\begin{tabular}{|c|c|c|c|}
\hline TOTAL & 326 & & \\
\hline Mer Egée, Macédoine et Asie Mineure & & 25 & $7,67 \%$ \\
\hline Aegyptus + Cyrenaeca & & 1 & $0,31 \%$ \\
\hline Afrique latine & & 9 & $2,76 \%$ \\
\hline Africa Proconsularis & 3 & & $0,92 \%$ \\
\hline Mauretania Tingitana & 1 & & $0,31 \%$ \\
\hline Numidia & 5 & & $1,53 \%$ \\
\hline Péninsule Ibérique & & 6 & $1,84 \%$ \\
\hline Hispania Baetica & 3 & & $0,92 \%$ \\
\hline Hispania Tarraconensis & 3 & & $0,92 \%$ \\
\hline Galliae, Germaniae, Britannia & & 104 & $31,90 \%$ \\
\hline Aquitania & 4 & & $1,23 \%$ \\
\hline Gallia Belgica & 11 & & $3,37 \%$ \\
\hline Gallia Lugdunensis & 23 & & $7,06 \%$ \\
\hline Gallia Narbonnensis & 5 & & $1,53 \%$ \\
\hline Germania Inferior & 33 & & $10,12 \%$ \\
\hline Germania Superior & 26 & & $7,98 \%$ \\
\hline Britannia & 2 & & $0,61 \%$ \\
\hline Provinces du Danube et Dalmatia & & 40 & $12,27 \%$ \\
\hline Noricum & 2 & & $0,61 \%$ \\
\hline Raetia & 10 & & $3,07 \%$ \\
\hline Dacia & 5 & & $1,53 \%$ \\
\hline Dalmatia & 10 & & $3,07 \%$ \\
\hline Moesia Inferior & 2 & & $0,61 \%$ \\
\hline Pannonia Inferior & 3 & & $0,92 \%$ \\
\hline Pannonia Superior & 8 & & $2,45 \%$ \\
\hline Provinces des Alpes & & 0 & $0,00 \%$ \\
\hline Sardinia & & 1 & $0,31 \%$ \\
\hline Italie (sans Rome et Ostia-Portus) & & 56 & $17,18 \%$ \\
\hline Italia regio 01, Latium et Campania & 13 & & $3,99 \%$ \\
\hline Italia regio 02, Apulia et Calabria & 3 & & $0,92 \%$ \\
\hline Italia regio 03, Bruttium et Lucania & 1 & & $0,31 \%$ \\
\hline Italia regio 04, Samnium & 1 & & $0,31 \%$ \\
\hline Italia regio 05 , Picenum & 2 & & $0,61 \%$ \\
\hline Italia regio 06, Umbria & 3 & & $0,92 \%$ \\
\hline Italia regio 07, Etruria & 5 & & $1,53 \%$ \\
\hline Italia regio 08 , Aemilia & 5 & & $1,53 \%$ \\
\hline Italia regio 10 , Venetia et Histria & 12 & & $3,68 \%$ \\
\hline Italia regio 11, Transpadana & 11 & & $3,37 \%$ \\
\hline Urbs Roma & 71 & 71 & $21,78 \%$ \\
\hline Ostia + Portus & 13 & 13 & $3,99 \%$ \\
\hline
\end{tabular}




\section{Etymologie, genèse et variations du vocabulaire}

L'étymologie de negotium et ses dérivés ne pose pas des problèmes. Negotium est la négation de otium et peut indiquer toute activité de nécessité. D'après Benveniste, le mot était formé par imitation du grec $\sigma \xi \mathrm{o} \lambda \Leftrightarrow \alpha$ et s'est rapproché pour son sens économique au cours de son évolution à $\pi \rho \gamma \mu \alpha$, ce qui a permis la formation des dérivés negotiari ( $\pi \rho \alpha \gamma \mu \alpha \tau \varepsilon(\varepsilon \sigma \psi \alpha \mathrm{l})$ et negotiator $\left(\pi \rho \alpha \gamma \mu \alpha \tau \varepsilon(\tau \eta \omega)^{6}\right.$.

Le mot negotiari fait son apparition au début du $\mathrm{I}^{\mathrm{er}}$ siècle a.C. dans les inscriptions latines ou bilingues des cités grecques pour indiquer des communautés de Romains et d'Italiens qui negotiantur installées dans ces cités. Les inscriptions les plus anciennes viennent de Délos et datent de 88 ou 87 a.C. ${ }^{7}$. Peu après, Cicéron reprend le mot dans les Verrines et l'introduit (?) dans la littérature latine en y ajoutant le nom d'agent negotiator ${ }^{8}$. L'usage courant qu'il en fait dans cette oeuvre montre qu'à cette époque déjà les mots n'avaient plus besoin d'explication. Le participe présent negotians est utilisé comme alternative pour le nom d'agent negotiator pour la première fois par Cicéron dans une lettre à Atticus de 51 a.C.. C'est un usage qui deviendra courant dans les inscriptions de l'époque impériale, mais qui demeure rare dans la littérature ${ }^{9}$. Deux lettres de Cicéron écrites respectivement en 46 et en 45 a.C. attestent pour la première fois le mot negotiatio ${ }^{10}$.

Contrairement au grec $\pi \rho \alpha \gamma \mu \alpha \tau \varepsilon(\varepsilon \sigma \psi \alpha \mathrm{l}$, le mot negotiari et ses dérivés negotiator et negotiatio ont une signification exclusivement économique ${ }^{11}$, mais ce ne sont pas les seuls mots dérivés de negotium qui jouent un rôle dans le langage des affaires. Des expressions verbales, telles que negotium/-a agere, gerere, exercere, conficere, contrahere etc. sont souvent utilisées dans un sens économique comme alternatives pour le mot negotiari. Seuls 232 passages (47\%) sur les 490 sélectionnés utilisent un ou plusieurs mots dérivés de negotiari. Ainsi, les textes littéraires et juridiques étalent une grande variété dans l'expression des affaires et des hommes d'affaires.

Le vocabulaire des inscriptions par contre est très monotone. 320 inscriptions des 326 $(98 \%)$ utilisent un ou plusieurs mots dérivés de negotiari. $279(86 \%)$ attestent des negotiatores ou des negotiantes ${ }^{12}, 40$ (12\%) mentionnent negotiari, le plus souvent pour indiquer un conventus de Romains/Italiens qui negotiantur. Trois inscriptions (1\%) renvoient à une negotiatio (voir tableaux infra).

\footnotetext{
${ }^{6}$ Benveniste 1951 ; cf. Fevrier-Prévotat 1981, 369-370 ; cf. AE 1897, 12 ; Quint., Inst., 2.21 .3 ; 3.6.57-58 ; 7.1 .

${ }^{7}$ CIL, $1^{2}$, 830 ; 831. Cf. Hatzfeld 1919, 193-195 ; Nicolet 1966-1974, 358-363 ; Barlow 1978, 146-147.

${ }^{8}$ Cic., I Verr., 1.20 ; II Verr., $1.14 ; 20$; 68 ; 153 ; 2.6 ; 7 ; $15 ; 34 ; 73 ; 153$; 168 ; 188 ; $3.45 ; 96$; 137 ; $138 ; 4.43 ; 54 ; 93 ; 5,8 ; 140 ; 158 ; 161$.

${ }^{9}$ Cic., Fam., 5.21 .10 : negavi me cuiquam negotianti dare (cf. Cic., Fam., 6.1 .4 ; 1.6 ; 2.8 ; 3.5). A part Cicéron je n'ai trouvé que huit passages chez d'autres auteurs où negotians est utilisé, dont deux fois avec la qualité d'un adjectif : Petr. 101.4 (dominus ... familiae negotiantis) 43.6 (homo negotians) ; Vell. 2.11.2 (per publicanos aliosque negotiantes) ; Sen., Ep., 36.5 ; 94.14 ; Vitr. 5.1 .8 ; Suet., Aug., 42.3 ; Dig. 47.11.6.pr (Ulpianus).

${ }^{10}$ Cic., Fam., 6.8 .2 ; 13.66 .2 (vetus negotiatio Asiatica).

${ }^{11}$ Sauf bien sûr quand ils sont utilisés de façon métaphorique, cf. par exemple Ps.-Var., Sent., 61 ; 76.

${ }^{12}$ Il n'est pas possible de différencier parce que le mot est souvent abrégé ou mutilé.
} 


\section{Les significations matérielles du vocabulaire des negotia}

Le mot negotium a une signification très large. Il est évident que dans le domaine économique il peut être utilisé pour indiquer toute activité dans n'importe quel secteur (rural, commercial, financier, etc.). Mais ce n'est pas le cas pour negotiari et ses dérivés, sur la signification précise desquelles le désaccord continue de régner.

\section{Mercatura}

La majorité des attestations des mots negotiator, negotians et negotiari à l'époque impériale renvoient sans aucun doute aux marchands et au commerce. Environ trois quarts des inscriptions qui mentionnent des negotiatores comportent une spécification qui indique le produit commercialisé par les negotiatores en question, telle que negotiatores sagarii, vestiarii, vinarii, etc. ${ }^{13}$. Une inscription de Pouzzoles mentionne les mercatores qui ... negotiantur. Chaque fois que nous pouvons identifier les activités d'un negotiator ou de plusieurs à l'époque impériale dans les textes littéraires, ils apparaissent comme des négociants ${ }^{14}$.

Ces données semblent contraster avec les données de la fin de la république. A peu près tous les negotiatores républicains dont les activités peuvent être identifiées étaient des financiers privés. A l'inverse, aucun financier connu de l'époque impériale n'est jamais désigné expressément comme negotiator ou comme qui negotiatur ${ }^{15}$. (cf. infra)

Rougé y voit un glissement de sens. A la fin de la république les mercatores auraient été des marchands, tandis que les negotiatores auraient été des manieurs d'argent. Ensuite, ces deux catégories se seraient rapprochées au début de l'époque impériale jusqu'à ce que negotiator aussi puisse signifier un marchand en gros, tandis que mercator indiquait plutôt le marchand plus modeste. Il avouait que parfois les negotiatores pouvaient s'engager dans le commerce (comme p.e. Rabirius Postumus), mais selon lui «(les véritables affaires commerciales) ne représentent pas l'essentiel de (leurs) activités ${ }^{16}$. Kneissl a repris cette idée en y ajoutant deux passages de Cicéron qui semblent distinguer les negotiatores et les mercatores. Dans le discours Pro Plancio 64 Cicéron dit qu'en tant que questeur de Sicile il se comportait comme comis envers les negotiatores et iustus envers les mercatores. Dans les Verrines Cicéron demande qui était ce mystérieux 'Verrucius', qui selon les livres de compte des publicani avait prêté tant d'argent par l'intermédiaire des sociétés de publicains : mercator an negotiator an arator an pecuarius ... ${ }^{17}$.

Pourtant, Rougé et Kneissl avouent que les expressions telles que cives Romani / Italici qui negotiantur comprennent à la fois les mercatores et les faeneratores et banquiers.

\footnotetext{
${ }^{13}$ Pour des exemples cf. Inscriptiones Urbis Romae Latinae, index verborum VI, p. 4166-4167; CIL, XIII.1911; $1954 ; 8568$; CIL, III.5800.

${ }^{14}$ CIL, X, 1797. Cf. Apul., Met., 1.5 ; 13-14 ; Dig. 14.5.8.pr (Paulus); 18.1.71 (Paulus) ; 18.6.20.pr (Hermogenianus); 20.4.21.1 (Papinianus) ; 34.9.1.pr (Marcianus); 50.6.6(5).3 (Callistratus) ; Plin., Nat., 6.88 ; 146 ; 149 ; 12.88 ; 18.225 ; Suet., Cl., 18.2 ; Nero, 32.3 ; Tac., Ann., 13.51 ; Mart. 10.87.

${ }^{15}$ La théorie de Ernesti que les negotiatores jusqu'à l'époque augustéenne étaient des faeneratores ou des marchands de blé a été refutée par Hatzfeld il y a longtemps (Hatzfeld 1919, 194-195), mais continue de surgir (p.e. Bürge 1987, 494).

${ }^{16}$ Rougé 1966, 278, 280-282.

${ }^{17}$ II Verr. 2.188. Kneissl 1983, 73-75.
} 
Ainsi, Rougé et Kneissl distinguent les activités pratiquées par le negotiator des activités comprises dans le negotiari. C'est à mon avis peu probable, car cela impliquerait que la signification du nom d'agent de negotiari (negotiator) a été d'abord restreinte à la finance pour ensuite, à peine un demi-siècle plus tard, changer de sens et assumer une signification depuis toujours incluse dans le terme negotiari, mais prétendument exclue jusqu'alors de son nom d'agent. Le passage du Pro Plancio s'explique facilement par la différence de respectabilité ou par la différence entre les marchands en gros et les marchands de détail. L'énumération dans l'invective de Cicéron contre Verres à laquelle Kneissl fait référence est atypique. Normalement, Cicéron distingue trois grandes catégories économiques : les negotiatores, les publicani et les aratores / pecuarii ${ }^{18}$. En II Ver., 2.168, Cicéron dit que les negotiatores, les aratores et la communis Sicilia avaient érigés des statues en honneur de Verres et il ajoute la question rhétorique quod est aliud in illa provincia genus hominum? Nullum.

Si il est vrai qu'il existe peu de sources qui spécifient les activités commerciales de negotiatores à l'époque républicaine, les textes qui spécifient des activités financières ne sont ni très nombreux, ni sans ambiguïté. Le cas fameux des Salamini montre que le terme negotiator pouvait désigner un financier, mais rien ne permet d'en déduire que le terme à cette époque exclue les activités commerciales. Cicéron refusait des praefecturae à M. Scaptius et $\mathrm{P}$. Matinius parce qu'il avait décidé de n'en donner aucune à tous ceux qui étaient negotiatores dans sa province de Cilicie. Il semble étrange qu'il ait consenti de donner des praefecturae aux marchands en gros, surtout parce que les marchands en gros avaient aussi les moyens de pratiquer l'usure. Le cas de T. Herennius doit être nuancé. Bien qu'il fût un negotiator ex Africa qui faisait la banque (argentariam fecit) à Leptis Magna, il ne s'ensuit pas qu'il était negotiator parce qu'il était argentarius. Rien n'exclut que Herennius se soit transformé en marchand après avoir été banquier ou d'avoir pratiqué les deux professions parallèlement. Cicéron le présente comme victime de la férocité de Verres contre les négociants qu'il faisait jeter en prison, torturer et exécuter et dont il confisquait la cargaison sous prétexte qu'ils étaient des pirates alliés de Sertorius ${ }^{19}$. Une lettre à Atticus (2.16.4) écrite en 60 a.C., fait référence à un conflit entre publicani et negotiatores concernant les droits de douane, où il ne fait aucun doute que les negotiatores étaient des marchands.

Bref, bien qu'il soit vrai que les negotiatores et ceux qui negotiantur attestés dans l'œuvre de Cicéron aient été souvent et surtout des manieurs d'argent, il n'y a aucune raison pour en conclure que la signification de negotiator à cette époque excluait les mercatores. Les negotiatores mentionnés par Cicéron sont très proches de l'aristocratie. Comme c'était le cas en Europe préindustrielle le sommet du monde d'affaires à Rome était la finance. C'est à cela que nous devons imputer l'importance de la faeneratio dans les negotia privata attestés dans l'œuvre de Cicéron et le contraste avec les negotiatores infiniment plus modestes dans les inscriptions.

\footnotetext{
${ }^{18}$ Cf. Cic., II Verr. 2.7 ; 153 ; Font., 12 ; 15 ; 32 ; Clu., 198 ; Flac., 38 ; 73 ; Pis., 98 ; Scaur., 35.

${ }^{19}$ Cic., II Verr. 1.14 ; 5.155-156 ; Andreau 1987, 419-420, 427-428 ; pour les Herennii cf. Deniaux 1979.
} 


\section{Faeneratio - res argentaria}

Les negotiatores dans l'œuvre de Cicéron sont donc surtout actifs dans la finance ${ }^{20}$. Ainsi, les hommes de pailles dont s'est servi Brutus pour gérer ses faenera en Orient sont appelés des negotiatores. Les dettes qui écrasaient les Gaulois sous Fonteius étaient le fait de prêts accordés par les cives Romani qui negotiantur. En Gaule, aucun nummus n'aurait été dépensé sans passer par les livres de comptes des citoyens romains qui negotiantur. Sittius faisait preuve de son negoti gerendi studium quand il pratiquait l'usure comme faenerator - intermédiaire ${ }^{21}$.

La faeneratio n'implique pas forcément la res argentaria (la banque de dépôt). Mais il ne fait aucun doute que les argentarii furent inclus dans les communautés de Romains émigrés qui negotiantur depuis le début du dernier siècle a.C.. Il semble donc probable qu'à l'origine le mot negotiator pouvait désigner un argentarius et que negotiari pouvait couvrir le fait d'argentariam exercere. Mais la situation à l'époque impériale est moins claire. Nous ne connaissons aucun negotiator qui soit indubitablement un argentarius ou un nummularius. Aucun texte non plus ne nous présente des argentarii / nummularii qui negotiantur. L'inscription fameuse de la Porta Argentaria distingue les argentarii des negotiantes boari. Une autre inscription de Rome distingue les argentarii des exceptores itemque negotiantes vini Supernatium et Ariminium ${ }^{22}$. Mais est-il une coïncidence que dans ces deux cas les negotiantes reçoivent des spécifications qui excluent d'autres activités commerciales telles que la banque ? Une inscription de Cologne de c. 200 p.C. mentionne un negotiator nummularius. Andreau propose de lire negotiator (et) nummularius. Mais les expressions du type negotiator + spécification sont très communs et cette lecture serait unique ${ }^{23}$. L'argument que l'adjectif nummularius est très rare ne vaut pas grand chose, puisque les spécifications de negotiator / negotians que nous retrouvons dans les inscriptions ne sont pas toujours des $\operatorname{adjectifs}^{24}$.

Toutefois, il demeure remarquable qu'à part peut-être de ce negotiator nummularius les spécifications fréquentes dans les inscriptions qui accompagnent la désignation de negotiator / negotians ne font jamais référence à la banque ou au prêt d'argent. A la fois pourtant, la banque professionnelle n'échappe pas à la portée de la terminologie du negotiari. Bien qu'Ulpianus distingue l'administration d'une banque (argentaria) des negotia, Labeo utilise le terme negotiatio pour indiquer l'entreprise d'un argentarius. L'expression negotiationem exercere peut indiquer n'importe quelle entreprise, y compris celle d'un banquier professionnel ${ }^{25}$.

\footnotetext{
${ }^{20}$ cf. Hatzfeld 1919, 197-212 ; Nicolet 1966-1974, 362.

${ }^{21}$ Cic., Att., 5.21 .10 ; 6.1 .4 ; Font. 11 ; Sul. 58.

${ }^{22}$ CIL, VI, 31232 (= 1035); CIL, VI, 1101.

${ }^{23}$ CIL, XIII, 8553 ; cf. Andreau 1987, 217-218. Il n'y aucune raison de croire que les manticularii negotiatores mentionnés en CIL, XIII, 6797 et (peut-être) 7222 étaient des manieurs d'argent plutôt que des producteurs et des négociants de bourses et de petits sacs, cf. Kneissl 1983, 82-84 ; Andreau 1987, 247-249.

${ }^{24}$ CIL, XI, 1621 ; XIII, 8354. Pour nummularius comme adjectif cf. Dig. 14.3.20 (Scaevola) : mensae nummulariae. Peut-être le negotiator nummularius de Cologne était littéralement un marchand qui s'occupait de l'approvisionnement de la région en monnaies de bronze ; cf. Reynolds \& Volk 1990, 388390 ; contra Van Heesch 2004, 247-258.

${ }^{25}$ Dig. 5.1.19.1 (Ulpianus) ; Dig. 2.13.6.3. (Labeo chez Ulpianus) ; Dig. 14.3.11.3 (Ulpianus).
} 
La situation pour les faeneratores à l'époque impériale est aussi brouillée. Valère Maxime utilise le mot negotiatio pour indiquer l'entreprise du faenerator, mais comme nous venons de le voir negotiatio a toujours gardé le sens général propre au negotiari, de telle sorte qu'il est impossible d'en déduire que les faeneratores étaient aussi des negotiatores ou que le negotiari inclut le faenerari ${ }^{26}$. Quand les Gaulois se révoltèrent en 21 p.C. parce qu'ils étaient écrasés par les dettes ils attaquèrent en premier lieu les negotiatores romains, qui semblent donc avoir pratiqué toujours l'usure ${ }^{27}$. Mais, est-ce qu'ils étaient des negotiatores parce qu'ils pratiquaient le prêt à intérêt, ou étaient-ils des négociants qui pratiquaient aussi l'usure? Sénèque distingue le faenerans du $n_{\text {negotians }}{ }^{28}$. Selon Modestinus il était illégal pour un gouverneur et ses adjudants de negotiari ou de pratiquer le prêt à intérêt, ce qui semble indiquer que le dernier n'était pas compris dans le premier ${ }^{29}$.

La faeneratio et les faeneratores - très mal vus dans la culture romaine - sont absents de la documentation épigraphique. Bien que le prêt à intérêt fût pratiqué à tous les niveaux sociaux et économiques, de celui du prêteur sur gage à celui du sénateur financier et des spécialistes intermédiaires, il n'existe aucune inscription connue qui mentionne un faenerator ${ }^{30}$. S'ensuit-il que les inscriptions érigées pour et par les faeneratores se taisent sur la nature de leur profession ou est-ce que les faeneratores se présentent dans les inscriptions comme des negotiatores sans spécification? Un épigramme de Martial pourrait l'indiquer : Et delator es et calumniator, et fraudator es et negotiator, et fellator es et lanista, miror quare non habeas, Vacerra, nummos. Ce passage ressemble à des passages semblables chez Plaute et Sénèque le Père contre les faeneratores $^{31}$.

\section{Industrie et services}

Un témoignage frappant qui indique que le negotiator pouvait être aussi un producteur, est fourni par les inscriptions qui mentionnent des negotiatores artis alicuius (e.g. artis cretariae, artis lapidariae, artis prossariae etc. ${ }^{32}$. Il n'est pas permis pourtant de conclure de l'absence de artis qu'un negotiator n'était pas aussi le producteur des produits dont il faisait le commerce. L'addition de artis était avant tout une mode régionale limitée aux provinces du Nord-Ouest ${ }^{33}$. L'inscription funéraire du negotians materiarius P. Alfius Erastus de Florentia montre plusieurs outils utilisés dans

\footnotetext{
${ }^{26}$ Val. Max. 4.8.3.

${ }^{27}$ Tac., Ann., 3.42.

${ }^{28}$ Sen., Ep., 94.14 .

${ }^{29}$ Dig. 12.1.33 (Modestinus).

${ }^{30}$ Cf. Verboven 1993, 80-82.

${ }^{31}$ Mart. 11.66. Cf. Plaut., Curc., 505-509; Sen., Contr. 9.1.12.

32 Selon Schlippschuh 1974, 58 artis désigne la qualité supérieure des produits commercialisés. C'est invraisemblable : ars désigne normalement la faculté ou le savoir technique de pratiquer une activité et non pas les produits ou les articles qui en sont le résultat. Il serait très naturel de dire ars negotiandi, mais ce n'est pas ce que disent les inscriptions, où ars est le plus souvent lié avec un adjectif dérivé d'un substantif : ars cretaria, ars clostraria, ars ratiaria etc. Notez que artis dans ces inscriptions est au singulier. Cf. le cas du negotiator cervesarius artis offecturae de AE 1928, $183=1941$, 168, où artis est liée avec offecturae. La signification en est obscure, mais il est difficile de le mettre en rapport avec autre chose que la production ou la conservation de bière.
}

${ }^{33}$ Cf. Kneissl 1983, 80. 
l'exploitation des carrières et la préparation des pierres. Sur un de ceux-ci nous voyons clairement les lettres P(ublius) A(lfius) E(rastus). De telles inscriptions sont bien connu de l'instrumentum domesticum et font référence au propriétaire de la manufacture où les outils furent fabriqués ou - dans ce cas-ci - utilisés ${ }^{34}$. Le negotiator pistoricius Tertinius Secundus, Nervien d'origine, mais résident de Cologne c. 200 p.C., était sans doute le propriétaire d'un pistrinum plutôt qu'un marchand de farine ${ }^{35}$. Plusieurs tuiles de l'époque de Septime Sévère trouvées à Rome portent des cachets qui mentionnent explicitement les negotiatores impliqués dans leur fabrication ${ }^{36}$. Dans les Digestes aussi, nous retrouvons plusieurs attestations de negotiari et ses variantes appliqués à l'industrie ou aux services. Gaius considère chaque type de artificium vulgare exercere comme une forme de negotiari. Scaevola applique les expressions negotiari et negotia exercere à n'importe quelle profession ${ }^{37}$. Helvius Successus, le père de l'empereur Pertinax, aurait exploité une negotiatio lanaria, appelée ailleurs dans le texte taberna coactiliaria $^{38}$.

Pourtant, dans le cas de negotiatores producteurs, ce n'est sans doute pas la production en soi qui leur confère la qualité de negotiator, mais le fait qu'ils ont investi leur argent dans une entreprise dont ils commercialisaient ensuite les produits.

Negotiari semble aussi avoir eu une signification plus large que mercari en ce qui concerne le type de 'produits' vendus. Ainsi, negotiari pouvait comprendre la spéculation en bien-fonds. Une inscription de Herculanum atteste d'un sénatus-consulte visant la spéculation immobilière, stipulant que si quelqu'un achetait un bâtiment negotiandi causa pour le détruire et revendre le terrain plus cher, il serait condamné à payer le double au fisc ${ }^{39}$. Les services aussi pouvaient entrer dans le domaine du negotiari. Une inscription d'Arles fait mention d'un negotiator familiae gladiatoriae d'origine peut-être syrienne mais actif dans la Narbonnaise ${ }^{40}$.

\section{Publicains}

Presque toutes les énumérations connues distinguent les negotiatores des publicani ${ }^{41}$. Ceci n'exclut évidement pas que des publicains s'engageaient dans les negotia privata ou que les sociétés développaient des negotia privata ${ }^{42}$, mais leur qualité de negotiator ou de qui negotiatur ne dérive pas de leur qualité de publicanus. Ainsi, Cicéron mentionne deux catégories de citoyens romains dans la province d'Asie : aut ...

\footnotetext{
${ }^{34}$ Kneissl 1983, 79 ; CIL, XI, 1620 ; cf. la nouvelle édition Epigraphica, 1975, p. 213 - 217.

${ }^{35}$ CIL, XIII, 8338 = RSK 307.

${ }^{36}$ Le formulaire est toujours composé de trois rubriques : les figlinae où l'object était fabriqué, le domaine où les figlinae se situaient et le nom du negotiator. Cf. CIL, XV, $415 ; 417 ; 418 ; 419 ; 430 ; 431 ; 649$; 879. Cf. $A E$ 2000, 831 a-b : tuiles fabriquées pour la legio XX Valeria Victrix en Britannia par un A. Viducius, peut-être membre de famille du negotiator Britannicianus L. Viducius Viduci f. Placidus (AE 1977, 0512 = AE 1983, 643 et AE 1975, 651).

${ }^{37}$ Cf. Dig. 14.4.1.pr.1-2 (Ulpianus) ; 15.1.27.pr (Gaius) ; 37.14.18.pr (Scaevola) ; 38.1.45 (Scaevola).

${ }^{38}$ SHA, Pert., 1.1 ; 3.3-4.

${ }^{39}$ CIL, X, 1401.

${ }^{40} C I L$, XII, 727. Wierschowski 2001, 234, n 308. Cf. AE 2000, $210 ; 270 ; C I L$, III, $7343=6113$.

${ }^{41}$ Cf. Cic., Flac., 38 ; Font., 12 ; Pis., 98 ; Scaur., 35 ; Att., 2.16.4.

42 cf. p.e. Cic., II Verr., 2.170.
} 
publicani sunt ... aut ... ita negotiantur ut locupletes sint. Velleius Paterculus distingue les publicani des alii negotiantes ${ }^{43}$.

Pourtant le cas des publicains est probablement à interpréter de façon restreinte, limité aux membres des societates vectigalium, qui jouissaient d'une personnalité juridique. Beaucoup de taxes provinciales, comme les dîmes de Sicile étaient affermées sur place aux hommes d'affaires individuels ou réunis en societas 'privée'. La même chose est vrai pour l'affermage des taxes municipales. Les activités des conductores ou redemptores ayant des contrats avec l'Etat pour la prestation de services ou la livraison de produits entrent sans doute dans le negotiari. Ainsi, les marchands de blé et d'autres produits destinés à l'annona à l'époque impériale sont appelés des negotiatores dans les textes littéraires et juridiques ${ }^{44}$.

\section{Agriculture et élevage}

La frontière entre l'agricultura et la negotiatio n'est pas toujours rigide. Dans les expressions du type cives Romani qui (aliquo loco) negotiantur, le verbe negotiari comprend toutes les activités économiques possibles, y compris les activités rurales. Mais ces expressions datent sûrement d'une époque où les Romains d'outre mer étaient surtout des marchands et des banquiers ou financiers. Il est vrai bien sûr que les negotiatores dans les provinces pouvaient acquérir des domaines et qu'ils étaient souvent en plus des aratores / pecuarii, mais il ne s'ensuit pas que leur qualité de negotiatores dérive de ces activités rurales ${ }^{45}$.

Le concept même de negotia est souvent opposé à la vie rurale. Ainsi Horace proclame le bonheur de celui qui procul negotiis ... paterna rura bobus exercet suis ${ }^{46}$. Si nous considérons les énumérations et classifications dans lesquelles des negotiatores et le negotiari sont comprises, nous voyons qu'ils sont à peu près toujours rigoureusement distingués des agricolae / pecuarii ${ }^{47}$. Les rares exceptions qui semblent suggérer que les agricolae / pecuarii pouvaient être inclus dans la 'classe' des negotiatores et que negotiari pouvait comprendre arare / pascere sont ambiguës. Dans le discours Pro Plancio, Cicéron distingue les negotiatores, les mercatores, les mancipes et les socii. (Planc. 64) Les aratores n'y sont pas mentionnés, bien qu'il s'agisse de l'envoi de grain sicilien à Rome, mais cela peut s'expliquer aussi parce que les aratores n'étaient que de simples contribuables. Dans le discours Pro Fonteio, Cicéron énumère les catégories diverses de citoyens romains en Gaule transalpine : publicani, agricolae, pecuarii, ceteri negotiatores. Brunt interprète le mot ceteri comme inclusif : 'and the rest of the negotiatores'. Mais dans ce cas on s'attendrait plutôt à reliqui au lieu de ceteri, ainsi

\footnotetext{
${ }^{43}$ Cic., Q. fr., 1.1.6 ; Vell. 2.11.2.

${ }^{44}$ cf. p.e. Suet., Aug., 42.3 ; Dig. 50.6.6(5).3-8 (Callistratus).

45 Pour le caractère mi-officiel cf. Nicolet 1966-1974, 358-363. Cf. Zoumbaki 1998-1999 pour les éleveurs et agriculteurs qui negotiantur. Cf. Vennonius qui laissaient des nomina, res et possessiones (Cic., Fam., 13.72).

${ }^{46}$ Hor., Epod. 2.1-4 ; cf. aussi Cic., Clu., 198 : qui in agro Larinati praedia, qui negotia, qui res pecuarias habent.

${ }^{47}$ Cf. par exemple Cic., II Verr., 2.153 ; 168 ; 188 ; Font., 12 ; Bell. Afr., 36.2.
} 
qu'il vaut probablement mieux interpréter ceteri dans un sens exclusif ${ }^{48}$. Columella parle du rusticari uel nauigare uel etiam genere alio negotiari ${ }^{49}$. Mais, genere alio peut renvoyer exclusivement à nauigare. Ailleurs, Columella oppose les deux façons de gagner la vie. Dans l'énumération fameuse sur les genera amplificandi reliquendique patrimonii la negotiatio est opposée à l'agricolatio ${ }^{50}$. Dans le Satiricon de Petronius, le parvenu Chrysanthus - sans doute un affranchi - fut un homo negotians qui avait fait fortune dans la viticulture. Mais, ce n'est pas clair si Chrysanthus était un propriétaire de vignobles, ou seulement un negotiator qui achetait la vendange avant la récolte des raisins ${ }^{51}$. Selon Festus le nom des Numidas, qui étaient des nomades vivant de leur bétail, leur fut donné parce que id genus hominum pecoribus negotiatur. Ici, negotiari semble comprendre à la fois l'élevage et le commerce du bétail. ${ }^{52}$

Un autre passage de Columella aide à mieux comprendre la relation entre l'agriculture et les negotia. Columella prévient de ne pas permettre au vilicus de negotiari pour soimême ou d'investir l'argent du maître dans (l'achat) d'animaux ou d'autres choses à revendre, car une telle negotiatio n'est pas compatible avec la cura uilici. Le passage est repris vers la fin de l'œuvre où il est dit qu'une telle activité ferait de lui plutôt un negotiator qu' un agricola ${ }^{53}$. Il s'ensuit que l'arare / pascere n'est pas comprise dans le negotiari, mais que la frontière entre negotiari et arare / pascere peut être facilement traversée parce que l'essentiel du negotiari est le développement d'activités commerciales, peu importe que ce soit avec des produits agricoles ou autres. Ainsi, le terme negotiari pouvait indiquer les activités d'un propriétaire marchand qui s'oriente vers un marché et qui s'occupe lui-même de la commercialisation de ses produits agricoles. C'était le cas sans doute de beaucoup d'Italiens émigrés, membres des communautés de Romains qui negotiantur.

Pourtant, il est rare que de tels cas soient spécifiés. La majorité de propriétaires de terres ne s'engageaient probablement pas dans la commercialisation de leurs produits. Comme Pline le Jeune, ils vendaient leurs vendanges et les produits de leurs domaines à des negotiatores. En plus, l'agriculture constituait une activité honorable, tandis que le commerce était plutôt déshonorant. Un propriétaire qui s'engageait dans la commercialisation de ses produits (personnellement ou par le biais de ses esclaves ou de ses affranchis), se serait présenté tout au plus comme le maître d'esclaves ou le patron d'affranchis negotiatores, mais non pas comme negotiator lui-même. C'est ainsi que nous connaissons quelques esclaves negotiatores, tel que par exemple Flavianus L. Aemilii qui se désigne comme negotiator et dispensator de son maître, ou Venustus servus vicarius de Musicus, esclave de l'empereur Claudius ou de $\mathrm{Nero}^{54}$.

\footnotetext{
${ }^{48}$ Brunt 1988, 168-170. cf. Voir aussi Clemente 1974, 114-115 ; D'Arms 1981, 26. Mais cf. Suet., Prata fr. 176 : reliquos et ceteros ; reliquos dicimus relictos ex omnibus, ceteros quos excipimus ; Cf. Cic., Pis., 98 : ... quem tribuni militares, quem reliqui milites ...

${ }^{49}$ Col. 12. praef. 2.

${ }^{50}$ Col. 12. praef. 8.

${ }^{51}$ Petr. 43.4. Cf. Bergh 2002. Comparez peut-être M. Inthatius Vitalis negotiator vinarius à Lugdunum, honoré à Alba (en Narbonnaise) connus pour ses vins de qualité : CIL, XIII, 1954 ; Wierschowski 2001, 327-328, n 454.

${ }^{52}$ Fest., p. 173 (éd. Lindsay).

${ }^{53}$ Col. 1.8.13 ; 11.1.24.

${ }^{54}$ CIL, VI, $3687=C I L$, VI, 30902 (Flavianus) ; CIL, VI, 5197 (Venustus).
} 


\section{La théorie d'Andreau}

Negotiari et ses dérivés ont probablement été formés comme néologismes inspirés du mot grec $\pi \rho \alpha \gamma \mu \alpha \tau \varepsilon\left(\varepsilon \sigma \psi \alpha \iota\right.$ au début du I ${ }^{\mathrm{er}}$ siècle a.C. dans la région de la mer Egée. Selon Andreau l'aspect géographique aurait dans un premier temps prévalu. Ainsi, negotiari auraient indiqué les activités de citoyens romains ou d'Italiens romanisés émigrés pour pratiquer des affaires privées de n'importe quelle nature. Puis, à l'époque augustéenne ou triumvirale la dimension géographique aurait disparu et l'application aurait été limitée au commerce et aux commerçants ${ }^{55}$.

Il est vrai que la première attestation de negotiatores actifs en Italie ne se retrouve qu'un demi siècle après la formation du mot negotiari. Mais la documentation républicaine est problématique parce qu'elle se compose surtout des textes de Cicéron, qui fournit presque $90 \%$ de tous les passages pertinents (146 sur un total de 163). Dans l'œuvre de Cicéron les mots negotiari et negotiator sont toujours utilisés dans des contextes provinciaux, mais il ne s'agit pas forcément toujours de citoyens émigrés hors de l'Italie. Plusieurs fois dans ses lettres à Atticus concernant l'affaire du prêt de Brutus à Salamis, Cicéron répète qu'il avait refusé par principe d'accorder des praefecturae à des negotiatores ayant des affaires dans sa province de Cilicia. Il avait appliqué cette règle aux procurateurs de Brutus, M. Scaptius et P. Matinius, à M. Laenius - un ami d'Atticus et de L. Manlius Torquatus - et à C. Vennonius - un ami de Cicéron lui-même - mais il avait fait une exception pour deux autres procurateurs de Brutus, un autre M. Scaptius et un L. Gavius, parce qu'ils opéraient en Cappadocia et non pas en Cilicia. Ces personnes étaient des chevaliers romains, qui entretenaient de bons contacts avec des sénateurs proéminents. Ils conduisaient des affaires importantes dans les provinces, mais ils ne semblent pas s'y être installés de manière permanente ${ }^{56}$. C'est aussi le cas pour P. Sittius, qui possédait des terres en Campanie tout en pratiquant l'usure dans les provinces et les royaumes clientélaires ${ }^{57}$.

La documentation épigraphique concernant les negotiatores et ceux qui negotiantur de l'époque républicaine se compose presque exclusivement d'inscriptions érigées par des communautés de Romains ou d'Italiens qui negotiantur établies en dehors de l'Italie. Il est évident que de telles communautés ne se retrouvent pas en Italie ${ }^{58}$. Pourtant, ces inscriptions montrent aussi que l'identité romaine ou italienne n'était pas inhérente au concept de negotiari, car sinon on n'aurait pas eu besoin de préciser que ces negotiatores ou ces qui negotiantur étaient des cives romani ou des italici. A Délos le mot negotiari est appliqué à des hommes d'affaires grecs ${ }^{59}$.

Il n'y a pas d'inscription connue de l'époque républicaine qui mentionne explicitement un negotiator ou un groupe de qui negotiantur en Italie. Mais étant donné la rareté

\footnotetext{
55 Andreau 2000.

${ }^{56}$ Cic., Att., 5.21.10-13 ; 6.1.4-8 ; 1.14 ; 2.7-9 ; 3.5-7. Sur les personnages voir Nicolet 1966-1974, 896897 (Gavius) ; 922 (Laenius) ; 1014 (les Scaptii) ; Deniaux 1993, 235-236, 509-511 (Laenius), 473-474 (Vennonius).

${ }^{57}$ Cic., Sul., 56-59 ; Nicolet 1966-1974, 308, 361.

${ }^{58}$ Bien qu'elles pouvaient offrir une inscription en Italie, cf. $A E 1900,178=C I L, \mathrm{I}^{2}, 836$. Cf. les

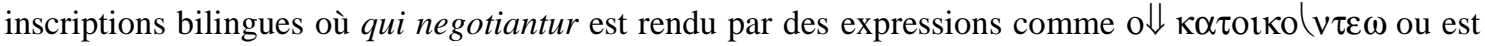
simplement non traduit. Cf. p.e. CIL, III, $743 ; 1210$.

${ }^{59} A E$ 1905, $36=C I L$, III, 7240.
} 
d'inscriptions républicaines cette absence n'est pas nécessairement significative. La première inscription qui mentionne expressément des negotiatores (et non des qui negotiantur) date de l'époque triumvirale et vient de Rome même. Elle atteste des negotiatores ex area Saturni à Rome ${ }^{60}$. Est-il une coïncidence que la première inscription qui utilise le mot negotiator ne vient pas d'une communauté de Romains installés en provinces, mais de Rome même?

Si il est vrai que le mot negotiari a été formé par analogie avec le mot grec $\pi \rho \alpha \gamma \mu \alpha \tau \varepsilon(\varepsilon \sigma \psi \alpha$, qui signifie tout simplement (entre autre) 'pratiquer des affaires' sans aucune connotation géographique, comment expliquer que les Romains aient d'abord ajouté une limitation géographique pour ensuite, un demi siècle plus tard, la retirer? Cela dit, il est bien sûr possible que l'origine grecque du mot negotiari lui ait donné une couleur provinciale ou exotique, faisant en sorte qu'il était plus facilement utilisé dans des contextes provinciaux, mais il ne s'ensuit pas que l'émigration hors de l'Italie soit impliqué dans les termes.

\section{Le negotiari et l'économie de marché}

Il n'y a qu'un critère solide qui demeure pour circonscrire l'application de negotiari / negotiator : l'engagement et l'investissement dans une entreprise commerciale de n'importe quelle nature. C'est le monde du marché qui caractérise le negotiator et le negotiari. Tout ce qui peut entrer dedans peut venir dans l'emprise du negotiari. Si Chrysanthus avait été un propriétaire de vignobles, il aurait été viticulteur comme Pline le Jeune, mais seul Chrysanthus l'aurait été en tant que homo negotians parce que contrairement à Pline il s'occupait aussi de la commercialisation de son vin. A l'époque républicaine, negotiari et ses dérivés couvraient tous les domaines de l'économie commerciale. Ce n'est qu'après, au début de l'époque impériale, que l'usage est devenu plus restreint par l'exclusion des banquiers et des faeneratores.

Ainsi, dans le cas d'un legs d'esclaves qui faisait exception pour les negotiatores, Labeo était d'opinion que tous les esclaves préposés à l'exploitation d'une entreprise de n'importe quelle nature (praepositi negotii exercendi causa) étaient exclus, tandis que les esclaves personnels (comme les cubicularii ou les obsonatores) ou les esclaves préposés à la supervision d'autres esclaves travailleurs, comme par exemple des pêcheurs, étaient inclus ${ }^{61}$.

C'est ainsi que negotiari exprime mieux que n'importe quel autre mot en latin l'unité profonde du champ du marché et du commerce. L'infâme Scaptius, chevalier romain, ami et homme de paille de Brutus, le banquier et marchand T. Herennius, citoyen romain de Syracuse et banquier à Leptis Magna, C. Apronius Raptor, marchand de vin et armateur installé à Lyon et decurio à Trèves, les négociants qui rendent grâce à la déesse Nehalennia, tous s'identifient comme des negotiatores, c'est-à-dire comme des spécialistes du marché.

\footnotetext{
${ }^{60} \mathrm{CIL}, \mathrm{I}^{2}, 810$.

${ }^{61}$ Dig. 32.1.65 (Marcianus) Legatis seruis exceptis negotiatoribus ...
} 


\section{Les significations sociales du vocabulaire des negotia}

Le langage des negotia est structuré selon deux axes différents. Le premier exprime la distance symbolique entre l'acteur et ses actes, le deuxième exprime la relation et la distance entre l'auteur et son sujet. Le premier axe met en cause la personnalité et l'identité du sujet. Il distingue les signifiants d'identité, d'activité et de possession, qui créent une distance symbolique croissante entre l'acteur et ses affaires, qui est à son minimum dans le cas où l'identité sociale coïncide avec les affaires. A ce point l'engagement de l'acteur est tel que sa personne s'efface devant ses affaires et qu'il devient lui-même l'objet de ses propres actes, l'homme devient un 'homme d'affaires', negotiator ou negotians. La distance symbolique s'accroît quand la personne se détache de ses actes et devient acteur reconnaissable, un qui negotiatur ou quelqu'un qui negotia exercet. La distance est à son maximum au point où l'acte s'efface devant la personne, quand l'acteur devient possesseur et maître de ses affaires. Ainsi, l'expression negotia habere et les signifiants de possession en général peuvent servir à éloigner le sujet honorable de ses intérêts déshonorants et à souligner l'engagement indirect et passif, tandis que l'expression negotiari et les autres signifiants d'activité indiquent l'engagement direct et actif.

Le deuxième axe mesure différents degrés de spécificité et de relation entre l'auteur et son sujet. Nous distinguons ici l'attribution à des sujets anonymes de l'attribution à des sujets spécifiques. Cette dernière catégorie est elle-même divisée entre des sujets réels contemporains, des sujets réels mais dans un passé plus ou moins éloigné et des sujets fictifs.

Ainsi, un negotiator connu par son inscription funéraire entre dans la catégorie des personnes spécifiques réelles proches. Le Vergilius de Horace qui est désigné comme negotiator par Acron entre dans la catégorie des personnes réelles historiques, parce que du point de vue de Acron ce Vergilius était trop loin éloigné dans le temps ${ }^{62}$. Trimalcion dans celle des personnes spécifiques fictives. Les negotiatores dans les expressions telles que Italici qui negotiantur ou qui figurent dans les énumérations nombreuses qui les mentionnent à côté d'autres groupes sociaux, entrent dans la catégorie de personnes anonymes.

Les deux axes forment une matrice dans laquelle le vocabulaire des negotia économiques est structuré. En tant que "structure structurée", cette matrice traduit les réticences de l'idéologie aristocratique vis-à-vis l'engagement commercial; en tant que "structure structurante" il permet à l'auteur de redistribuer les acteurs économiques selon l'éthique aristocratique, sans pour cela affecter leur liberté d'agir ${ }^{63}$.

\footnotetext{
${ }^{62}$ Hor., Carm., 4.12.

${ }^{63}$ Sur les concepts "structure structurée" et "structure structurante" voir Bourdieu [1972] 2000.
} 


\begin{tabular}{|c|c|c|c|}
\hline DEGRE DE SPECIFITE & $\begin{array}{l}\text { Signifiants } \\
\text { d'identité }\end{array}$ & Signifiants d'activité & $\begin{array}{l}\text { Signifiants de } \\
\text { possession }\end{array}$ \\
\hline & $\begin{array}{l}\text { Negotiator } \\
\text { Negotians }\end{array}$ & $\begin{array}{l}\text { Negotiari } \\
\text { Negotium/-a gerere } \\
\text { Negotium/-a exercere } \\
\text {... }\end{array}$ & $\begin{array}{l}\text { Negotia habere } \\
\text { Negotia }+ \text { gen. }\end{array}$ \\
\hline Sujets anonymes & & & \\
\hline Sujets fictifs & & & \\
\hline Sujets spécifiques dans un passé éloigné & & & \\
\hline $\begin{array}{l}\text { Sujets spécifiques proches de l'auteur / } \\
\text { locuteur }\end{array}$ & & & \\
\hline
\end{tabular}

Les effets de cette structure immanente au langage se manifestent dans la répartition des différents types de sources, qui sont distribués dans la matrice de manière très hétérogène.

\begin{tabular}{|c|c|c|c|c|c|c|}
\hline & \multicolumn{3}{|c|}{ Inscriptions } & \multicolumn{3}{|c|}{ Textes } \\
\hline TOTAL & 326 & $100 \%$ & & 490 & $100 \%$ & \\
\hline $\begin{array}{l}\text { dérivés de negotiari } \\
\text { - } \quad \text { negotiator, negotians, negotiari } \\
\text { - } \quad \text { negotiatio } \\
\text { autres expressions avec negotium/-a }\end{array}$ & \begin{tabular}{|l|}
320 \\
318 \\
3 \\
6 \\
\end{tabular} & $\begin{array}{l}98,16 \% \\
97,55 \% \\
0,92 \% \\
1,84 \% \\
\end{array}$ & $\begin{array}{r}\mathbf{1 0 0 \%} \\
99,38 \% \\
0,94 \%\end{array}$ & $\begin{array}{l}232 \\
183 \\
59 \\
258 \\
\end{array}$ & $\begin{array}{l}47,35 \% \\
37,35 \% \\
12,04 \% \\
52,65 \% \\
\end{array}$ & $\begin{array}{r}\mathbf{1 0 0 \%} \\
78,88 \% \\
25,43 \%\end{array}$ \\
\hline $\begin{array}{l}\text { negotiator ou negotians } \\
\text { negotiator } \\
\text { negotians } \\
\text { negotiari }\end{array}$ & 279 & $\begin{array}{r}85,58 \% \\
/ \\
1 \\
12,27 \%\end{array}$ & $\begin{array}{r}87,19 \\
/ \\
/ \\
12,50 \%\end{array}$ & $\begin{array}{l}111 \\
102 \\
9 \\
78\end{array}$ & $\begin{array}{l}22,65 \% \\
20,82 \% \\
1,84 \% \\
15,92 \% \\
\end{array}$ & $\begin{array}{r}47,84 \% \\
43,97 \% \\
3,88 \% \\
33,62 \% \\
\end{array}$ \\
\hline \multicolumn{7}{|c|}{ NEGOTIARI - NEGOTIATOR - NEGOTIANS } \\
\hline Negotiari - negotiator - negotians & 318 & $100 \%$ & & 183 & $100 \%$ & \\
\hline $\begin{array}{l}\quad \text { Signifiants d'identité } \\
\text { negotiator spécifique contemporain } \\
\text { negotians spécifique contemporain } \\
\text { negotiator/negotians spécifique } \\
\text { contemporain }\end{array}$ & $\begin{array}{l}279 \\
/ \\
230\end{array}$ & $\begin{array}{l}87,74 \% \\
/ \\
/ \\
72,33 \%\end{array}$ & $\begin{array}{l}100 \% \\
/ \\
/ \\
82,44 \%\end{array}$ & $\begin{array}{l}7 \\
1 \\
8\end{array}$ & $\begin{array}{l}60,66 \% \\
3,83 \% \\
0,55 \% \\
4,37 \%\end{array}$ & $\begin{array}{l}\mathbf{1 0 0 \%} \\
6,31 \% \\
0,90 \% \\
7,21 \%\end{array}$ \\
\hline $\begin{array}{l}\text { negotiator non spécifique } \\
\text { negotians non spécifique } \\
\text { negotiator/negotians non spécifique }\end{array}$ & $\begin{array}{l}/ \\
/ \\
50\end{array}$ & $\begin{array}{l}\text { / } \\
/ \\
15,72 \%\end{array}$ & $\begin{array}{l}\text { / } \\
\text { / } \\
17,92 \%\end{array}$ & $\begin{array}{l}82 \\
7 \\
89\end{array}$ & $\begin{array}{l}44,81 \% \\
3,83 \% \\
48,63 \%\end{array}$ & $\begin{array}{l}73,87 \% \\
6,31 \% \\
80,18 \%\end{array}$ \\
\hline $\begin{array}{l}\text { negotiator personnage fictif } \\
\text { negotians personnage fictif } \\
\text { negotiator/negotians personnage } \\
\text { fictif }\end{array}$ & I & $\begin{array}{l}1 \\
1 \\
0 \%\end{array}$ & $\begin{array}{l}1 \\
1 \\
0 \%\end{array}$ & $\begin{array}{l}10 \\
1 \\
11\end{array}$ & $\begin{array}{l}5,46 \% \\
0,55 \% \\
6,01 \%\end{array}$ & $\begin{array}{l}9,01 \% \\
0,90 \% \\
9,91 \%\end{array}$ \\
\hline $\begin{array}{l}\text { negotiator personnage historique } \\
\text { negotians personnage historique } \\
\text { negotiator/negotians personnage } \\
\text { historique }\end{array}$ & 1 & $\begin{array}{l}1 \\
1 \\
0 \% \\
\end{array}$ & $\begin{array}{l}1 \\
1 \\
0 \% \\
\end{array}$ & $\begin{array}{l}3 \\
0 \\
3 \\
\end{array}$ & $\begin{array}{l}1,64 \% \\
0 \% \\
1,64 \% \\
\end{array}$ & $\begin{array}{l}2,70 \% \\
0 \% \\
2,70 \% \\
\end{array}$ \\
\hline $\begin{array}{l}\text { Signifiant d'activité (negotiari) } \\
\text { negotiari acteur non spécifique } \\
\text { negotiari acteur fictif } \\
\text { negotiari acteur historique } \\
\text { negotiari acteur contemporain }\end{array}$ & $\begin{array}{ll} & 40 \\
39 & \\
0 & \\
0 & \\
1 & \end{array}$ & $\begin{array}{r}12,58 \% \\
12,26 \% \\
0 \% \\
0 \% \\
0,31 \%\end{array}$ & $\begin{array}{r}\mathbf{1 0 0 \%} \\
97,50 \% \\
0 \% \\
0 \% \\
2,50 \% \\
\end{array}$ & $\begin{array}{l}59 \\
6 \\
1 \\
12\end{array}$ & $\begin{array}{l}42,62 \% \\
32,24 \% \\
3,28 \% \\
0,55 \% \\
6,56 \%\end{array}$ & $\begin{array}{l}\mathbf{1 0 0 \%} \\
75,64 \% \\
7,69 \% \\
1,28 \% \\
15,38 \%\end{array}$ \\
\hline
\end{tabular}


Le vocabulaire des inscriptions est très monotone. Sur un total de 326 inscriptions retenues, 318 (98\%) utilisent negotiator, negotians ou negotiari. La grande majorité de ces inscriptions (279 où $88 \%$ ) utilisent les signifiants d'identité negotiator ou negotians $^{64}$, dont $229(82 \%)$ sont attribuées à des personnes spécifiques contemporaines, et $50(18 \%)$ à des personnes non spécifiques (en général pour désigner des collegia). Le signifiant d'activité negotiari est présent dans 40 inscriptions (13\%). Une seule inscription - un carmen epigraphicum - utilise negotiari appliqué à une personne spécifique contemporaine ${ }^{65}$. Des 39 autres, 34 renvoient à un conventus de Romains, d'Italiens ou d'autres qui negotiantur. En général les inscriptions mentionnant ceux qui negotiantur sont des inscriptions honorifiques ou religieuses érigées par ceuxci. Les inscriptions parlant de negotiatores ou de negotiantes par contre montrent toute la diversité habituelle des inscriptions ${ }^{66}$.

Parmi les 490 passages des sources littéraires et juridiques où negotium, ou un ou plusieurs de ses dérivés sont utilisés dans un sens économique, seulement 232 (47\%) utilisent un dérivé de negotiari, dont 59 (12\%) passages qui parlent d'une negotiatio et 183 (37\%) passages qui utilisent negotiator, negotians, negotiari ou une combinaison des ces trois. De ces 183 passages 78 passages (43\%) utilisent le verbe negotiari, 111 passages (61\%) parlent de negotiatores (102 cas) ou de negotiantes (9 cas).

Cette plus grande variété du vocabulaire dans les textes littéraires et juridiques n'est pas étonnant en soi, mais les autres différences sont plus significatives. Le verbe negotiari est presque 3 fois plus fréquent dans les textes littéraires que dans les inscriptions (34\% comparée à 13\%), mis à part son participe présent negotians, qui est beaucoup plus fréquent dans les inscriptions que dans les textes. Il est possible qu'à l'origine negotians s'est introduit dans le vocabulaire des inscriptions pour des raisons semblables que celles qui sont sous-jacentes à la préférence relative dans les textes littéraires. Mais, il se transforme très vite en synonyme de negotiator, c'est à dire en un substantif qui désigne une profession.

Dans les sources littéraires les signifiants d'identité appliqués à des personnes spécifiques sont très rares. Sur les 111 passages qui parlent de negotiatores ou de negotiantes seulement 8 passages $(7 \%)$ les utilisent pour indiquer des personnes spécifiques contemporaines. 5 de ces 8 passages traitent de l'affaire du prêt usuraire de Brutus à Salamis, où Cicéron désigne quelques hommes d'affaires de manière assez implicite comme des negotiatores ou des negotiantes ${ }^{67}$. Les trois autres passages viennent de l'In Verrem et parlent de T. Herennius negotiator, de C. Servilius vetus negotiator et d'un certain C. Verrucius - un personnage fictif mais prétendu réel dont Cicéron veut savoir qui il était : mercator an negotiator an arator an pecuarius ${ }^{68} .89$ passages $(80 \%)$ utilisent les termes negotiator ou negotians dans un sens non

\footnotetext{
${ }^{64} 166(60 \%)$ de ces 279 parlent de negotiator, 63 (23\%) de negotians Dans les autres cas le mot est abrégé (neg. ou negot.) ou seulement partiellement conservé.

${ }^{65}$ CIL, VI, $9659=$ CIL, VI, 33814. Notez CIL, XIII, $5221=$ AE 1998, $978:[---$ n]egotia[tores] $/ \ldots$ qui [negotiantur?].

${ }^{66}$ Inscriptions funéraires $61 \%$ (169); votives et religieuses 22\% (60); honorifiques 10\% (27).

${ }^{67}$ Cic., Att. $5.21 .10 ; 6.1 .4,6$; $2.8-9 ; 3.5$.

${ }^{68}$ Cic., II Ver., 2.188.
} 
spécifique, $11(10 \%)$ pour des personnes fictives, 3 (3\%) pour indiquer des personnes spécifiques mais historiques. Ainsi, dans les lettres de recommandation de Cicéron aucune des personnes recommandées n'est désigné comme negotiator, bien qu'il y en ait plusieurs qui sont désignés par des signifiants d'activité (negotiari, negotia exercere, ...) ou de possession.

Cette tendance peut être marquée aussi pour le verbe negotiari. Des 78 passages dans les sources littéraires et juridiques qui utilisent ce verbe 59 (76\%) l'utilisent pour des sujets non spécifiques, 1 (1\%) pour une personne historique, $6(8 \%)$ pour des personnes fictives et seulement $12(15 \%)$ pour des personnes spécifiques contemporaines. A première vue negotiari est donc plus populaire pour indiquer des personnes spécifiques que negotiator, mais il faut nuancer car l'oeuvre de Cicéron fournit 10 des 12 cas où une forme de negotiari est appliquée à une personne spécifique contemporaine ${ }^{69}$.

Des considérations sociales semblent se manifester plus clairement dans les sources littéraires. Je n'ai repéré aucun passage avec un signifiant d'activité ou d'identité appliqué à un sénateur. Cicéron utilise des signifiants d'activités - comme negotiari pour des chevaliers, mais les personnes en question sont plutôt obscures. Parfois, par un détour significatif, des signifiants d'identité et d'activité sont appliqués à des membres de statut élevé pour les exclure symboliquement de leur classe respectable. C'est le cas le plus claire pour C. Appuleius Decianus, à qui Cicéron dit : Quo usque negotiabere, cum praesertim sis isto loco natus $?^{70}$

Ces observations montrent que le langage des textes littéraires évite l'application de negotiari et ses dérivés à des personnes spécifiques. Au lieu du verbe negotiari les textes préfèrent des expressions verbales plus générales telles que negotia gerere ou exercere (qui peuvent avoir un sens plus général). Ainsi Cicéron parle du studium negoti gerendi de Sittius et de la magnitudo animi in negotiis gerendis de Rabirius Postumus ${ }^{71}$. Contrairement à negotiari et ses dérivés, ces expressions ne sont pas liées exclusivement à l'économie commerciale. Ce n'est donc pas une coïncidence si Cicéron utilise negotiari pour Decianus, mais studium negoti gerendi pour Sittius.

Selon Valencia Hernández la différence entre negotiator et negotiari dérive de la valeur identifiant du premier et celle masquant du dernier ${ }^{72}$. Je suis d'accord avec la valeur identifiante du terme negotiator (et de son équivalent negotians), mais je ne crois pas que l'expression verbale qui negotiatur était choisie pour masquer des choses. Negotiari signifie un engagement actif dans le commerce et/ou la finance, les deux étant également mal vues. On comprend mal comment Cicéron aurait pu objecter à Appuleius Decianus : Quo usque negotiabere, cum praesertim sis isto loco natus, si negotiari avait une valeur masquante.

L'intention de masquer, par contre, est bien présente dans le cas des expressions verbales avec negotium / - a (negotia exercere, gerere ...) qui ne partagent pas le caractère commercial de negotiari. Surtout les constructions negotia habere ou negotia

\footnotetext{
${ }^{69}$ Cic., Att. 6.1 .4 ; Fam. 12.26 .1 ; 13.17 .1 ; 22.1 ; 26.2 ; 43.1-2 ; 50.1 ; II Ver., 2.73 ; 5.161 ; Flac. $70-71$ (les autres sont Sen., Ep., 119.5 et Sal., Cat., 40.2).

${ }^{70}$ Cic., Flac., 70.

${ }^{71}$ Cic., Sul., 58 ; Rab. Post., 3.

${ }^{72}$ Valencia Hernández, 1989-1990, 211-212 ; cf. García Brosa 1999, 185 ; Brunt 1988, 170.
} 
+ génitif se prêtent bien à de telles intentions. Les negotia de Egnatius Rufus en Cilicia recommandés par Cicéron sont probablement des affaires financières, mais les recommandations ne les spécifient pas $^{73}$. Il est impossible de préciser la nature des negotia en Africa pour lesquelles Sex. Aufidius reçoit une recommandation de Cicéron. Nous savons seulement qu'il fut héritier de Q. Turius qui negotiatus est en Africa ${ }^{74}$.

A première vue, les inscriptions font preuve d'une préférence inverse pour les signifiants d'identité negotiator et negotians. Il faut être prudent avec de telles interprétations. La majorité des inscriptions qui nous intéressent sont des inscriptions funéraires, honorifiques ou votives, qui en général ont servies à présenter et qualifier des personnes, d'où la fréquence de signifiants d'identité de toutes sortes (fonctions remplies, parenté, ingénuité, ...). La grande majorité de telles inscriptions ne mentionnent aucune occupation ou engagement économique. Cela étant dit, pourtant, il est évident, qu'au moins en Italie et dans les provinces du Nord-Ouest la construction d'une identité sociale à partir d'un engagement dans les affaires commerciales, ne provoquait point des réactions négatives. Sinon, on comprendrait mal pourquoi même des personnes notables - des augustales, mais aussi des membres de l'ordo decurionum et même quelques chevaliers romains - sont assez régulièrement identifiés comme des negotiatores / negotiantes ${ }^{75}$.

On ne peut pas en tirer la conclusion qu'ailleurs les notables ne s'engageaient pas dans le commerce ou les finances. Pour établir le statut social des négociants les inscriptions sont peu appropriées, car la question ne porte pas sur le statut social de ceux qui se présentent comme des negotiatores ou des negotiantes, mais sur le nombre de ceux qui se présentent ou sont présentés comme des notables ou comme de simples citoyens, qui se sont engagés de façon active dans les negotia privata. C'est un problème qui a déjà été souligné par Sandra Joshel et que j'ai moi-même remarqué pour les procuratores privati. Ainsi, le fait qu'un pourcentage élevé de negotiatores / negotiantes connus par les inscriptions furent des affranchis n'implique pas que le monde des affaires était monopolisé par les affranchis, mais seulement que les affranchis étaient plus enclin de construire une identité sociale à travers leur occupation économique ${ }^{76}$.

Ce raisonnement vaut aussi pour les autres types de sources. Ainsi quand Cicéron énumère des catégories sociales en utilisant des signifiants d'identité de caractère non économique, il ne s'ensuit pas que les membres de ces catégories ne s'engagent pas dans le commerce et la finance. Quand il énumère par exemple tous qui connaissent bien les méfaits d'Appuleius Decianus : Sciunt haec omnes nobiles, sciunt boni viri, sciunt denique noti homines, sciunt mediocres negotiatores ${ }^{77}$, il ne s'ensuit pas que ces

\footnotetext{
${ }^{73}$ Cic., Fam., 13.43 ; 44 ; 45 ; 73 ; 74. Cf. Nicolet 1966-1974, 866-868 ; Deniaux 1993, 494-496.

${ }^{74}$ Cic., Fam., 12.26 ; 27 ; Nicolet 1966-1974, 794 ; Deniaux 1993, 458-460. Cf. les negotia de L. Aelius Lamia en Afrique, Cic., Fam., 12.29 .2 ; voir sur lui et ses affaires Nicolet 1966-1974, 762 ; Deniaux 1993, 439-441 Wiseman 1971, 202, 209 ; Rauh 1986, 16-17 ; Shatzman 1975, 288.

7539 notables : equites romani : CIL, III, 14927 ; VI, 29722 ; membres de l'ordo decurionum : CIL, XII, 1896 ; XIII, 2448 (= AE 1975, 616) ; VI, 33887 ; III, 1911 ; 11179 ; 2086 ; X, 5585 ; AE 1940, 64 ; decuriones ornamentarii : AE 1909, $81 ; 1900,203 ; 1974,123$ bis ; consessus cum ordine decurionum : CIL, XIII, 1954 ; augustales : CIL, IX, 5307 ; X, 1872 ; 3847 ; AE 1996, 416 ; seviri : CIL V, 5892 ; XI, $862 ; A E$ 1922, 82 ; seviri augustales : AE 1922, 82 ; 1982, 702 ; 2000, 632 ; 2000, 980 ; CIL, III, 5824 ; XIII, $1962 ; 1966 ; 2121 ; 6366 ;$ XIV, 397.

${ }^{76}$ cf. Joshel 1992, 60-61, 163-166 ; Verboven 2002, 255-256 ; Cristofori 2004, 96-103.

${ }^{77}$ Cic., Flac., 73.
} 
nobiles, boni viri et noti homines n'étaient pas actifs dans le commerce ou dans le finance, mais que leur qualité de notable fait obstacle à l'attribution de l'identité de negotiator.

\section{Mercator, mercari}

Les negotiatores étaient souvent des mercatores, mais les deux catégories ne se confondent pas. C'est sur cette relation difficile que la discussion sur la nature des negotiatores s'est portée le plus loin. Cicéron se disait comis negotiatoribus, mercatoribus iustus. De même l'énumération dans les Verrines : mercator an negotiator an arator an pecuarius ..., suggère une différence. Une inscription trouvée à Rome atteste un negotiator sagarius à côté d'un mercator sagarius ${ }^{78}$.

Cagnat y voyait la différence entre le marchand en gros et le marchand de détail ${ }^{79}$. Hatzfeld aussi pensait que le critère distinctif était la richesse et le volume des produits commercialisés, mais selon lui il y avait aussi un élément de subjectivité dans les termes qui pourrait expliquer pourquoi -dans les inscriptions - les mercatores préféraient se présenter comme des negotiatores. 'Le mercator est un marchand, avec la légère nuance de défaveur et comme le parfum de boutique qui s'attache à ce mot : le negotiator est « un homme qui est dans les affaires. »'. Ainsi, le negotiator n'était pas tant 'le négociant en gros que le gros négociant'. Cela pourrait expliquer par exemple pourquoi Cicéron se dit iustus envers les mercatores et comis des negotiatores ${ }^{80}$.

Baldacci partage en gros l'opinion de Hatzfeld, mais selon lui le prestige du negotiator diminue au cours du $\mathrm{I}^{\mathrm{er}}$ siècle p.C. faisant en sorte que negotiator et mercator deviennent largement des synonymes ${ }^{81}$. Garcia Brosa aussi croit reconnaitre une évolution sémantique, mais selon lui les mercatores devenaient de plus en plus riches et importants, effaçant ainsi, depuis l'époque augustéenne, la différence entre mercatores et negotiatores. Ainsi au I ${ }^{\mathrm{er}}$ siècle la seule différence qui aurait subsisté entre le terme negotiator et le terme mercator serait que negotiator pouvait aussi indiquer des faeneratores, tandis que mercator signifiait toujours un marchand ${ }^{82}$. Valencia Hernandez pense que les attestations rares de mercatores respectables ne sont que des figures littéraires ou des exceptions qui confirment la règle. A son avis, le mercator était toujours le petit marchand insignifiant, tandis que le negotiator était le marchand en $\operatorname{gros}^{83}$.

Qu'en est-il de toutes ces théories? Une différence est sûrement que le terme negotiator avait une signification plus large que le terme mercator. Même si en général les banquiers ne furent plus désignés comme des negotiatores, negotiari et ses dérivés ont gardé leur caractère diffus, tandis que le mercator est toujours resté un marchand sans plus, étant ni producteur, ni financier, ni spéculateur immobilier, ni fournisseur de services.

\footnotetext{
${ }^{78}$ Cic., Planc., 64; II Ver., 2.188 ; CIL, VI, 9675 ; VI, 12331. Cf. Hatzfeld 1919, 193. cf. Patterson 1998, 151-152.

${ }^{79}$ Cagnat 1904.

${ }^{80}$ Hatzfeld 1919, 195-196. Cf. Nicolet 1966-1974, 360-362.

${ }^{81}$ Baldacci 1967, 273-276.

${ }^{82}$ García Brosa 1999.

${ }^{83}$ Valencia Hernández 1989-1990.
} 
Mais cette différence matérielle n'explique pas tout. Est-ce qu'il est vrai que le mot mercator était évité parce que la mercatura fut considérée comme 'sordide'? Dans le fameux passage sur les artificia et quaestus liberales / sordidi dans le De Officiis, Cicéron utilise un critère quantitatif pour distinguer la mercatura respectable avec celle qui fut sordide, mais il n'utilise pas les mots negotiator ou negotiari ${ }^{84}$. Dans les Verrines, Cicéron parle des mercatores honesti et locupletes de Pouzzoles, ce qui semble suggérer que le mot mercator était applicable à des négociants riches et respectables, mais il note aussi qu'il y avait des liberti parmi eux ${ }^{85}$. Inversement, dans le discours Pro Valerio Flacco Cicéron parle des mediocres negotiatores à côté des nobiles, des boni viri et des noti homines ${ }^{86}$. Quintilien parle de la merx sordida du negotiator $^{87}$. Mais ces deux passages opposent les negotiatores et leurs activités aux notables et à la rhétorique. Il est évident que de ce point de vue les negotiatores aient été 'vulgaires' et leur profits 'sordides'. Martial compare le negotiator avec un delator, un calumniator et un fraudator $^{88}$. Mais il satirise et c'est bien possible - comme nous avons vu - qu'il envisage surtout la faeneratio, plutôt la mercatura. Les negotiatores attestés dans les inscriptions ne furent sûrement pas tous des 'gros négociants' ou des financiers riches. La majorité de ces inscriptions sont des inscriptions funéraires en somme assez modestes, émanant de marchands ayant peut-être une entreprise florissante, mais n'ayant sûrement pas les moyens financiers requis pour la faeneratio à grande échelle.

Dans la littérature latine, la fréquence des mots mercator et negotiator / negotians est à peu près égale. Dans les textes non bibliques du cdrom PHI\#5, mercator se rencontre 161 fois, dont 19 fois dans des textes de Plaute, Terence ou Caton l'Ancien, écrits bien avant la création du néologisme negotiari. Negotiator se rencontre 115 fois, negotians se rencontre 9 fois (pour 111 passages 'économiques'). L'application de mercator à des personnes spécifiques est encore plus rare que pour negotiator ou negotians. Je n'ai retrouvé que deux passages. Dans l'un Cicéron accuse Calventius, le père de L. Calpurnius Piso Caesoninus ${ }^{89}$, d'avoir été un mercator et praeco ; l'autre est celui que nous avons déjà rencontré concernant Verrucius. Mais, étant donné que 6 des 8 occurrences connues de negotiator appliqué à des personnes spécifiques contemporaines, traitent de la même affaire du prêt usuraire de Brutus à Salamis (cf. supra), il est claire qu'il n'y a pas lieu de conclure une préférence générale pour negotiator au lieu de mercator dans les textes littéraires et juridiques.

La situation est différente pour les inscriptions où les signifiants d'identité sont dominants. Mercator est presque 6 fois moins fréquent que negotiator ou negotians ${ }^{90}$.

\footnotetext{
${ }^{84}$ Cic., Off., 1.151.

${ }^{85}$ Cic., II Verr., 5.154.

${ }^{86}$ Cic., Flac., 73.

${ }^{87}$ Quint., Inst., 1.12.17.

${ }^{88}$ Mart. 11.66.

${ }^{89}$ Cic., Pis., fr. 11a.

${ }^{90}$ Pour les 51 attestations de mercator(es) dans la base de données de Manfred Clauss (EpigraphikDatenbank, Frankfurt-am-Main, Wolfgang Goethe Universität, Seminar für Alte Geschichte, http ://www.rz.uni-frankfurt.de/ clauss/index.html, ${ }^{\circ} 1996$ ) en avril 2004, et les indices des volumes VI, X et XI du CIL, (non encore inclus dans la base de données de Clauss à ce moment), il y a 320 inscriptions
} 
Dans la plupart des cas le sujet en question est une personne spécifique proche. Dans le registre de langue des inscriptions, il existait donc sûrement une préférence pour les termes negotiator / negotians au lieu de mercator. L'usage du mot mercator dans le nom du nobilissimum corpus mercatorum cisalpinorum et transalpinorum, qui réunît les plus importants négociants des routes transalpines semble témoigner de la respectabilité de la mercatura en question. Mais aucun membre connu du corpus n'est attesté par le mot mercator, tandis qu'au moins un d'eux est identifié comme negotiator ${ }^{91}$.

La question demeure pourquoi negotiari comporte la connotation d'intégration dans la communauté civique? Feuvrier Prévotat pense que la différence entre les dérivés de 'mercari' et les dérivés de 'negotiari' n'est pas d'ordre social ou économique, mais d'ordre sémiotique. Contrairement au mercator, la figure du negotiator aurait été fort liée à la communauté du peuple romain à l'étranger, où il entretenait des relations privilégiées avec les fonctionnaires romains. Les mercatores par contre étaient plus associés à une vie errante en dehors de la société civile telle qu'elle était conçue par les Romains $^{92}$. Il ne s'ensuit pas qu'à l'époque républicaine, les negotiatores furent toujours d'origine italienne. Déjà les inscriptions de Délos parlent de Graeci qui negotiantur et les inscriptions de l'époque impériale nous présentent plusieurs negotiatores pérégrins. Mais les mots de la famille de negotiator n'étaient pas contaminés par le sens de 'pérégrin' ou de l' 'Autre' propre à la famille de mercari. Ainsi, l'identité sociale du negotiator aurait comporté une connotation d'inclusion dans la société civile. Le mercator pouvait parfois jouir d'une certaine respectabilité, mais son identité sociale demeurait exclusivement économique.

\section{Ambiguïté et respectabilité}

Comme il a été remarqué par Feuvrier Prévotat, negotia et ses mots familles se caractérisent par leur polysémie et ambiguïté ${ }^{93}$. Loin de diminuer la signification pratique et la valeur informative de ces mots, c'est précisément cette ambiguité qui en augmente la signification. En désignant quelqu'un comme negotiator ou comme qui negotiatur la personne en question est classée dans une catégorie multiforme tant en ce qui concerne la nature de ses produits (rurales, artisanales, commerciales, financières, ...), qu'en ce qui concerne la nature de l'engagement (producteur, marchand en gros, financier, ...), et qu'en ce qui concerne son statut social. En plus, les textes littéraires suggèrent que le registre du negotiari peut facilement être substitué par celui plus honorables des expressions verbales renvoyant aux negotia (negotia exercere, gerere, etc.), ce qui à son tour a pu avoir une influence positive sur negotiari / negotiator.

Ainsi l'identité sociale du negotiator - contrairement à celle du mercator - est une identité assez large, définie par un engagement dans les affaires du marché mais sans aucune implication précise d'ordre social ou économique. Inhérente à cette identité est une liberté de choix qui est beaucoup plus grande que celle du mercator et qui permet

attestant des negotiatores ou des negotiantes. Curieusement par contre dans les inscriptions le nom propre Mercator est assez frequent.

${ }^{91}$ CIL, XIII, 2029. Sur le corpus mercatorum cisalpinorum et transalpinorum cf. Walser 1991.

${ }^{92}$ Feuvrier-Prévotat 1981. Cf. García Brosa 1999, 182-183 ; Valencia Hernández 1989-1990, 201.

${ }^{93}$ D'où peut-être que les énumérations qui sont par force précises, ne mentionnent pas les negotiatores. Cf. p. e. Cic., II Ver., 2.17. Dans Cic., II Ver., 2.168 les negotiatores sont considérés comme un genus hominum et non pas comme une ordo. 
une diversification d'intérêts économiques plus grande. Le negotiator a investi dans une entreprise commerciale, il peut - à la limite - aussi réinvestir ses profits ou sa fortune entière dans l'agriculture ou la politique.

La respectabilité du negotiator découle de cette liberté de choix. Le negotiator n'est pas un agriculteur, ni un publicain ou un notable, mais il peut le devenir. Ainsi la respectabilité du propriétaire terrestre ou du notable est à la portée du negotiator. Sa respectabilité n'est pas la respectabilité du notable dont la dignité découle du fait qu'il est censé être digne d'occuper des postes politiques ; c'est la respectabilité et le prestige de celui qui peut se transformer ou dont les enfants pourront se transformer en notables ou en propriétaires terriers ${ }^{94}$.

C'est à cela que Cicéron se réfère quand il fait une distinction entre la mercatura tenuis et la mercatura magna et copiosa en disant que la dernière mérite toute estime : si satiata quaestu vel contenta potius, ut saepe ex alto in portum, ex ipso portu se in agros possessionesque contulit ${ }^{95}$. L'estime ne dérive pas tant de l'importance en soi de cette mercatura pour l'approvisionnement (bien que cet aspect aussi peut jouer un rôle), mais par de la possibilité de se transformer.

Les mots de la famille de mercator ne comportent pas cette liberté de choix. Ainsi le mercator est avant tout celui qui n'a pas de choix, ou dont la liberté de choix n'est pas reconnue par l'auteur ou le locuteur. Il est vrai que Cicéron utilise parfois des expressions telles que mercatores locupletes et honesti, mais cette association entre mercator / mercari et des expressions d'honorabilité est extrêmement rare dans l'oeuvre de Cicéron et dans toute la littérature latine et les inscriptions. Certes, la mercatura peut se pratiquer de manière honeste, mais quand il s'agit d'identifier le marchand important, le terme de negotiator ou - dans les textes littéraires - des expressions verbales avec negotium / - $a$ sont préférées. Ainsi, ce n'est pas une coïncidence que l'usage de mercatores dans le passage de Cicéron est non spécifique. De même l'usage du terme mercator dans le titre du nobilissimum corpus mercatorum cisalpinorum et transalpinorum est non spécifique.

Ainsi, c'est la volonté d'évoquer la liberté de choix du sujet et son appartenance à une catégorie sociale en somme diffuse, qui peut expliquer la préférence dans les inscriptions pour le mot negotiator au lieu de mercator. Certes, la polysémie des termes peut cacher une réalité tout autre. Beaucoup de simples mercatores se sont sans doute appropriés le nom de negotiator, mais ils ne l'ont pas fait pour voiler leurs activités commerciales, mais pour s'investir de la respectabilité associée avec le nom de negotiator.

Koenraad Verboven Chercheur postdoctoral FRS - Flandres Université de Gand, Belgique

\footnotetext{
94 cf. Valencia Hernández 1989-1990, 195-196.

${ }^{95}$ Cic., Off., 1.151.
} 


\section{Bibliographie}

- Andreau, J. (1987) : La vie financière dans le monde romain. Les métiers de manieurs d'argent. (IV siècle av. J.-C. - III siècle ap. J.-C.), Bibliothèque des écoles françaises d'Athènes et de Rome 265, Rome.

- Andreau, J. (2000) : "Negotiator", Der Neue Pauly. Enzyclopädie der Antike, Bd. 8, Stuttgart, 783-785.

- Ausbüttel, Fr. M. (1982) : Untersuchungen zu den Vereinen im Westen des römischen Reiches, Frankfurter althistorische Studien 11, Kallmünz .

- Baldacci, P. (1967) : "Negotiatores e mercatores frumentarii nel periode imperiale", RIL 101, 273-291.

- Barlow, Ch. T. (1978) : Bankers, Moneylenders, and Interest Rates in the Roman Republic, Michigan.

- Benveniste, E. (1951) : "Sur l'histoire du mot negotium", ASNP 20, 21-25.

- Bergh, G. C. J. J. van den (2002) : "Legal Trouble Concerning a Vintage. Exegesis of D 19,1,25", in : Ligt 2002, 205-212.

- Ligt, L. de, J. de Ruiter, E. Slob, J. M. Tevel, M. Vrugt et L. C. van de Winkel, éds. (2002) : Viva Vox Iuris Romani. Essays in Honour of Johannes Emil Spruit, Amsterdam.

- Bourdieu, P. [1972] 2000 : Esquisse d'une théorie de la pratique. Précédé de Trois études d'ethnologie kabyle, Paris.

- Brunt, P. A. (1988) : "The Equites in the late Republic", in : Brunt 1988, 167-170.

- Brunt, P. A. (1988) : The Fall of the Roman Republic and Related Essays, Oxford.

- Bürge, A. (1987) : "Fiktion und Wirklichkeit. Soziale und rechtliche Strukturen des römische Bankwesens", ZRG 104, 465-558.

- Cagnat, R. (1904) : "Mercator", in : Daremberg \& Saglio, T. 3, p. 1731-1743.

- Clemente, G. (1974) : I Romani nella Gallia meridionale (II-I sec. a.C.). Politica ed economia nell'età dell'imperialismo, Il Mondo Antico. Studi di storia e di storiografia 2, Bologna.

- Cristofori, A. (2004) : Non arma virumque. Le occupazioni nell'epigrafia del Piceno, Tarsie. Studi di Antichistica 2, Bologna, 2 éd.

- D'Arms, J. H. (1981) : Commerce and Social Standing in Ancient Rome, Harvard.

- Deniaux, E. (1979) : "A propos des Herennii de la République et de l'époque d'Auguste", MEFRA 91, 623-650.

- Deniaux, E. (1993) : Clientèles et pouvoir à l'époque de Cicéron, Collection de l'école française de Rome 182, Rome.

- Feuvrier-Prévotat, Cl. (1981) : "Negotiator et mercator dans le discours cicéronien : essai de définition", DHA 7, 367-405.

- García Brosa, G. (1999) : “Mercatores y negotiatores : ¿Simples comerciantes?”, Pyrenae 30, 173-190.

- Hatzfeld, J. (1919) : Les trafiquants italiens dans l'Orient hellénistique, Bibliothèque des écoles françaises d'Athènes et de Rome 115, Paris.

- Joshel, S. (1992) : Work, Identity and Legal Status at Rome. A Study of the Occupational Inscriptions, Londres.

- Kneissl, P. (1983) : "Mercator-negotiator. Römische Geschäftsleute und Terminologie ihrer Berufe", MBAHG 2, 73-90.

- Nicolet, Cl. (1966-1974) : L'ordre équestre à l'époque républicaine (312-43 av. J.C.), 2 Vols., Bibliothèques des écoles françaises d'Athènes et de Rome 207, Paris. 
- Parkins H., et Chr. Smith, éds. (1998) : Trade, Traders and the Ancient City, Londres et New York.

- Paterson, J. J. (1998) : "Trade and Traders in the Roman World : Scale, Structure and Organisation", in : Parkins \& Smith 1998, 149-167.

- Rauh, N. (1986) : "Cicero's Business Friendships : Economics and Politics in the Late Roman Republic", Aevum 60, 3-30.

- Reynolds, J. et T. Volk (1990) : “Terence, Gifts, Curses, Cult and Society at Bath”, Britannia 21, 379-391.

- Rougé, J. (1966) : L’organisation du commerce maritime en méditerranée sous l'empire romain, Paris.

- Schlippschuh, O. (1974) : Die Händler im römischen Kaiserreich in Gallie, Germanien und den Donauprovinzen Rätien, Noricum und Pannonien, Amsterdam.

- Shatzman, I (1975) : Senatorial Wealth and Roman Politics, Collection Latomus 42, Bruxelles.

- Stuart, P. et J. E. Bogaerts (2001) : Nehalennia. Römische Steindenkmäler aus der Oosterschelde bei Colijnsplaat. 2 Bd., Leiden.

- Valencia Hernández, M. (1989-1990) : "Mercator y negotiator : ambigüedad y realidad económica en la obra de Cicerón", Caesaraugusta 66-67, 195-216.

- Van Heesch, J. (2004) : "Coins for the Army", in : Vermeulen et al. 2004, 247-258.

- Verboven, K. (1993) : "Le système financier à la fin de la république romaine", AncSoc. 24, 69-98.

- Verboven, K. (2002) : The Economy of Friends. Economic Aspects of Amicitia and Patronage in the Late Republic, Collection Latomus 269, Bruxelles.

- Vermeulen, Fr., K. Sas et W. Dhaeze, éds. (2004) : Archaeology in Confrontation. Aspects of Roman Military Presence in the Northwest (Studies in Honour of Prof. Em. Hugo Thoen), Archaeological Reports Ghent University 2, Gand.

- Walser, G. (1991), “Corpus mercatorum Cisalpinorum et transalpinorum”, $M H 42$, 169-175.

- Wierschowski, L. (2001) : Fremde in Gallien - 'Gallier' in der Fremde. Die epigraphisch bezeugte Mobilität in, von und nach Gallien vom 1. Bis 3. Jh. n. Chr., Historia Einzelschriften 159, Stuttgart.

- Wiseman, T. P. (1971) : New Men in the Roman Senate. 139 B.C. - A.D. 14, Oxford.

- Zoumbaki, S. (1998-1999) : "Die Niederlassung römischer Geschäftsleute in der Peloponnes", Tekmeria 4, 112-176. 